10 Questions

\title{
Ten questions concerning integrating smart buildings into the smart grid
}

\author{
Thomas M. Lawrence a, *, Marie-Claude Boudreau ${ }^{\text {b }}$, Lieve Helsen ${ }^{\text {c }}$, Gregor Henze ${ }^{\text {d }}$, \\ Javad Mohammadpour ${ }^{a}$, Doug Noonan ${ }^{e}$, Dieter Patteeuw ${ }^{c}$, Shanti Pless ${ }^{\mathrm{f}}$, \\ Richard T. Watson ${ }^{\mathrm{b}}$ \\ a College of Engineering, University of Georgia, USA \\ ${ }^{\mathrm{b}}$ Terry College of Business, University of Georgia, USA \\ ${ }^{c}$ Applied Mechanics and Energy Conversion Section, KU Leuven, Belgium \\ ${ }^{\mathrm{d}}$ Architectural Engineering at the University of Colorado - Boulder, USA \\ e Indiana University Public Policy Institute School of Public and Environmental Affairs, USA \\ ${ }^{\mathrm{f}}$ National Renewable Energy Laboratory, Golden, CO, USA
}

\section{A R T I C L E I N F O}

\section{Article history:}

Received 19 July 2016

Received in revised form

22 August 2016

Accepted 23 August 2016

Available online 27 August 2016

\section{Keywords:}

Smart grid

Smart buildings

Building control systems

Demand response

Energy policy

Thermal comfort

\begin{abstract}
A B S T R A C T
Recent advances in information and communications technology (ICT) have initiated development of a smart electrical grid and smart buildings. Buildings consume a large portion of the total electricity production worldwide, and to fully develop a smart grid they must be integrated with that grid. Buildings can now be 'prosumers' on the grid (both producers and consumers), and the continued growth of distributed renewable energy generation is raising new challenges in terms of grid stability over various time scales. Buildings can contribute to grid stability by managing their overall electrical demand in response to current conditions. Facility managers must balance demand response requests by grid operators with energy needed to maintain smooth building operations. For example, maintaining thermal comfort within an occupied building requires energy and, thus an optimized solution balancing energy use with indoor environmental quality (adequate thermal comfort, lighting, etc.) is needed. Successful integration of buildings and their systems with the grid also requires interoperable data exchange. However, the adoption and integration of newer control and communication technologies into buildings can be problematic with older legacy HVAC and building control systems. Public policy and economic structures have not kept up with the technical developments that have given rise to the budding smart grid, and further developments are needed in both technical and non-technical areas.
\end{abstract}

๑) 2016 Elsevier Ltd. All rights reserved.

\section{Introduction}

A growing challenge for the 21st century built environment is matching intermittent renewable electrical energy supply with variable demand in a world with:

- Increasing demand due to population growth and emerging developing economies

\footnotetext{
* Corresponding author. Driftmier Engineering Center, University of Georgia, Athens, GA, USA.

E-mail address: lawrence@engr.uga.edu (T.M. Lawrence).
}

- A growing fraction of intermittent electrical energy supply from renewable energy produced by solar photovoltaics and wind energy

- Concerns about the carbon emissions associated with burning fossil fuels.

Relevant statistics from the U.S. Department of Energy [1] and others include: (a) buildings consume approximately $40 \%$ of the world's total energy; and (b) heating, refrigeration and air conditioning (HVAC) systems represent about $50 \%$ of a typical building's total energy consumption. Thus, building HVAC systems represent roughly $20 \%$ of total global energy demand [1]. More recently, concern is not only directed to total energy consumption (kWh) but also to the current rate of consumption (kW) by a building. Organizations that operate a collection of buildings with a large 
combined electrical demand are prime candidates for participation in demand response programs. These include university or corporate campuses, medical centers, military bases, and large office complexes. Demand response can also be considered for other energy systems, such as a district chilled water cooling system.

Digital data streams (DDSs) [2] are a core innovation in the built environment that is enabling a revolution in management of both the electricity grid and building systems such as HVAC and lighting. A DDS is a continuous digital encoding and transmission of data describing the state of an entity, such as the electricity consumed by a piece of equipment or household, or the individual's social media posts. As more DDSs are created, grids and buildings can use the additional data to become smarter and make better decision about energy distribution and consumption. Smart meters are critical to the smart grid because they provide a DDS consumption stream, but they are just the beginning. There is also a need for DDS at every point of energy consumption and each point of production, such as a solar panel's current and predicted output over the next few hours. Futhermore, there is a need to go beyond smart meters to smart devices that can be controlled remotely to match current supply and demand.

The introduction of new technology creates new opportunities for managing electricity consumption and production more intelligently. It is an opportunity to provide new solutions to longstanding questions. This article addresses the wide range of complex issues and topics associated with using emerging technologies for integrating buildings and their systems into a smart grid.

\section{Ten questions concerning integrating smart buildings into the smart grid}

Smart buildings and the smart grid may not be perceived as having a direct impact on the indoor environment of buildings, but the interaction of smart buildings with a smart grid in areas like demand response programs can affect both the occupants' thermal comfort as well as the building's energy consumption (and related environmental impacts). The following questions with answers will help clarify key points about these interactions.

\subsection{What defines the "Smart Grid" and a "Smart Building"?}

\section{Answer:}

The smart grid is a broad term used in various contexts, and there have been many studies on the interactions between a smart grid and smart buildings [3]. The operative word here is the term "smart". In the context of physical systems such as a building or the grid, this term is generally used to define something that has advanced control systems and technologies that allow for interconnected operability giving the capability to operate efficiently in response to external and internal communications.

The smart grid is a modernized electrical grid using DDSs and information technology to more efficiently produce, transmit, and distribute electricity. Each sector of the electricity supply chain has different goals and objectives for the grid. The value and need for a smart grid may differ among various regions of the world. In some cases, the prime value is managing the integration of intermittent renewable energy supply. In other regions, it may be for peak load management, while still in other areas the focus may be on minimizing carbon emissions. The benefits for buildings are primarily cost minimization with minimal negative impact on operations. Buildings have the inherent potential to help manage the electrical energy demand on the grid. The sooner the integration of smart buildings with a smart grid is implemented, the more readily society will be able to manage an expanded renewable energy generation in the grid. Thus, in the long run the biggest beneficiary of this integration will be society as a whole.

The interaction of a collection of smart buildings (and other electrical energy users) with the smart grid can be thought of as a multi-agent system (MAS), which is characterized by a large number of interacting players. Individual local agents focus on their best interests, but they also interact with larger scale global agents such as the utility or system operator. Example publications for how MAS concepts work in this context are many. For example: in a distributed smart grid [4]; a survey paper for microgrid applications [5]; for how smart buildings and the smart grid interact [6]; and for energy use optimization in buildings, such as in Refs. [7,8].

Components of a smart grid include devices which allow for two-way communication between the utility or grid operator and the end users are: smart meters, grid management and demand (load) management software, information technology systems, building load or energy management systems and 'smart' end user equipment or appliances (Fig. 1).

The smart grid enables buildings to respond to and provide current operation data to grid operations. Smart buildings and their associated controls and equipment are capable of responding to demand response requests from the utility or system operator to manage peak demand to minimize demand charges or to adjust operations based on the real-time price of electricity. Some response measures may involve adjustments in HVAC operational setpoints (such as zone temperatures or supply air flow rates) or lighting, thus opening up potential occupant comfort perception problems (Fig. 2).

When done right, however, we postulate that a smart building can also provide a better indoor environmental quality (IEQ) for the building occupants. In this context, the important areas of IEQ affected include temperature, humidity, ventilation rates and lighting levels. Any demand response strategy for building control must still provide adequate ventilation rates to maintain acceptable indoor air quality as well as not lead to too high humidity levels in the space. ANSI/ASHRAE Standard 62.1-2016 [11] requires that relative humidity levels not exceed 65\% (unless higher levels are dictated by the space usage type) and also lists required ventilation rates to occupied spaces.

\subsection{What key technologies have enabled an efficient integration of smart buildings into a smart grid?}

\section{Answer:}

A combination of technological advances, market forces, electric utility system needs and governmental or other types of mandates are all paving the way for smart buildings and their associated equipment to be integrated with a smart grid. The primary development has been with the introduction of technology and protocols that allow for efficient and smooth communication of a building automation systems (BAS) with equipment and systems in the building, and just as importantly data exchange (DDSs) between the utility or system operator and the building with its associated equipment. Smart meters provide one, but not the only, way for this communication to occur.

One big step helping with this communication is the development of standard protocols that allow equipment from different vendors to interact with the smart grid; for example, the OpenADR specification, a fundamental part of the U.S. smart grid interoperability standards [12]. Communication between a building and the grid is only a first step; data must be able to be transmitted to/from the building automation and control systems. Building automation systems have evolved over the past $10-20$ years to allow greater control of energy use within a building in the form of building energy management systems (BEMSs). The integration of a smart building to the smart grid starts with a well-designed and well 


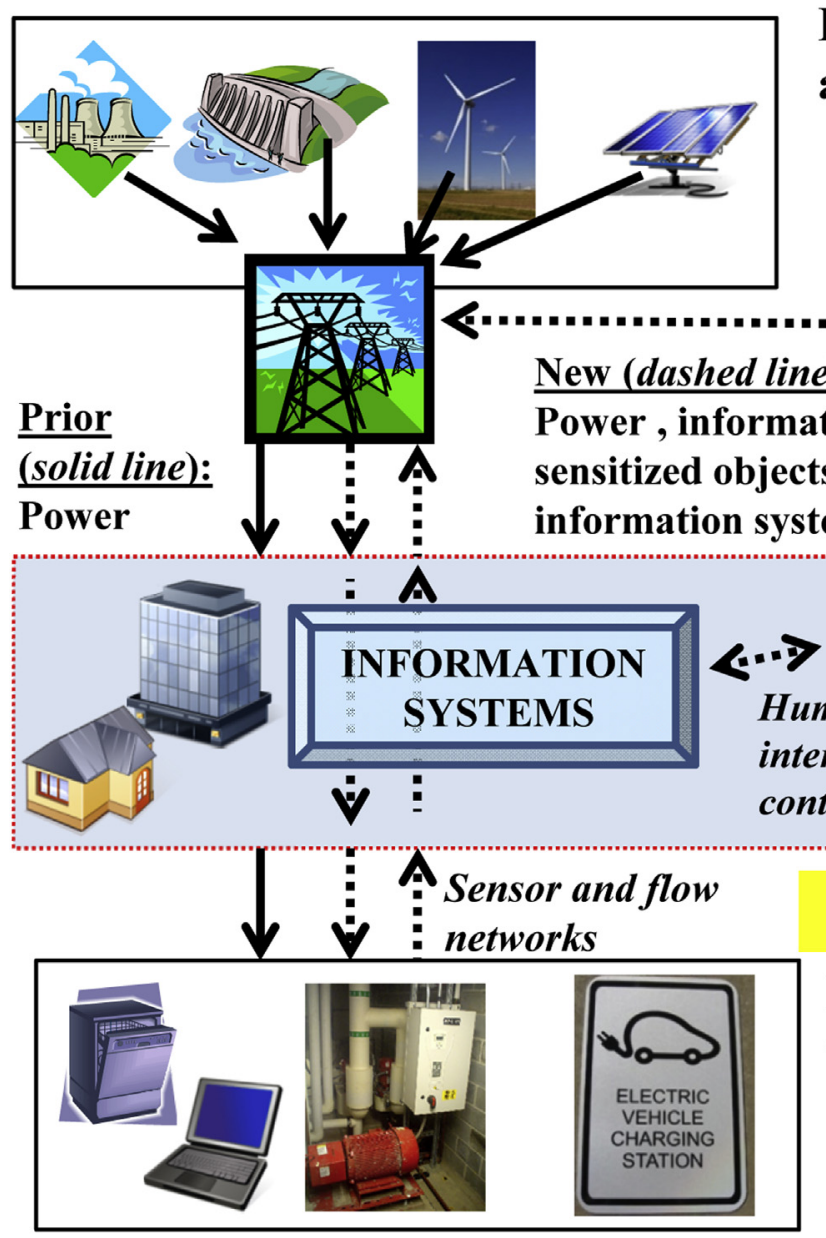

\section{Electricity generation, and the "smart-grid"}

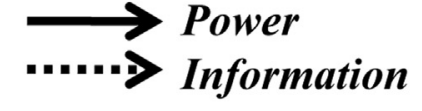

Fig. 1. The interaction of a smart grid and smart buildings in terms of energy and data flow.

managed BAS.

The development of web-enabled hardware and controllers provides another option for how a building's assembly of "Internet of energy things" will communicate with a smart grid. For example, in smaller scale buildings, such as a private residence, the hardware (e.g., a smart thermostat) may communicate directly with the grid either wirelessly or through smart metering. In larger scale buildings, however, there may be a need to manage overall control to optimize equipment operation through a BEMS. Lee [13] describes the need for BEMS evolution to account for emerging trends, such as a building potentially being a prosumer (a producer and consumer of electrical energy), the addition of electrical vehicle charging, etc. Development and maturation of building management and control systems is accelerating and these allow interaction with the smart grid, however, their development lags behind what is available on the utility side of the meter. One recent occurrence that will help here is the release of ASHRAE Standard 201-2016 (Facility Smart Grid Information Model) [14] that provides a common base for facilities to describe, manage and communicate electrical energy consumption and forecasts. This can be used to guide the evolution of existing facility energy management protocols.

Other non-technical developments are leading to the interaction of buildings and a smart grid. For example, in the 1980s some utilities began to offer financial incentives to customers to allow the utility to control some portion of their load for demand response. High-performance or "green" buildings are encouraged to include interaction with the smart grid via programs such as the U.S. Green Building Council's LEED rating system [15] and the International Green Construction Code [16].

Work continues on advancing the technology that drives a building and its equipment towards better connectivity and interoperability with the electricity provider. For example, communication protocols such as OpenADR may in the past have been viewed as only being suitable for large systems and facilities on larger computer platforms. But with the current state of technology, researchers are developing ways that even small, inexpensive computational devices suitable for adaptation in lower cost devices, such as an individual thermostat (residential or commercial), can be brought directly into the smart grid.

\subsection{What is the right scale in thinking about a smart grid as it interacts with buildings?}

\section{Answer:}

There are various relevant scales when considering the interaction with a smart grid: starting with a single building scale and moving into the electricity power grid scale (generation, transmission and distribution). The first scale to be considered is when the building is off-grid, in which case the grid must function as its own smart grid in balancing local electricity generation and demand. A review of the literature on off-grid systems with photovoltaic panels was done in Ref. [17].

However, for the more common case with a building connected 


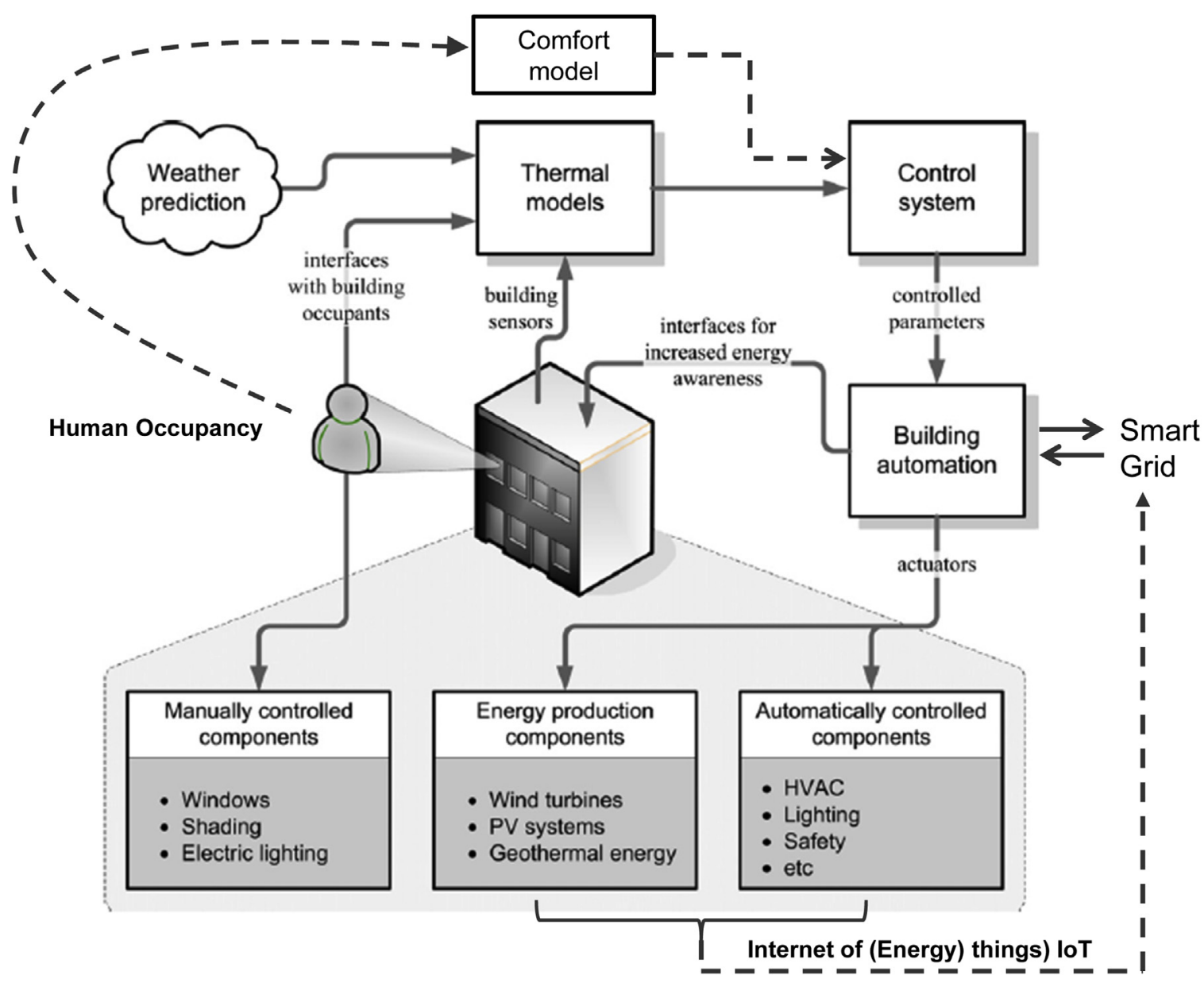

Fig. 2. Smart building component and connection to a smart grid (Adapted from Refs. $[9,10]$ ).

to an electricity grid, the right scale of thinking about interaction between a smart grid and buildings is first on the electricity power grid scale. The primary benefits of this interaction are described in Ref. [18]. Benefits include a reduction in peak electricity generation, transmission and distribution capacity, reduced stress on electrical sub-stations, and in managing the demand-supply balance in systems with a high share of intermittent renewable energy sources. Buildings can give flexibility to management of the electricity grid by reducing demand when requested. In order to quantify the potential gains due to this interaction, typical building-level studies simplify the electricity system to an electricity price profile. However as shown by Refs. $[19,20]$, these price profiles can potentially cause HVAC systems to show a significant (shifted) peak demand, even higher than the original peak demand, right before a price increase. Hence, the correct way of studying the aforementioned interaction is by evaluating and modeling the electricity grid and building systems simultaneously [21].

Practical implementation and associated gains depend on the magnitude of the electricity demand, the ability to smoothly adjust demand and availability of energy storage (potentially a major component in this interaction). Residential buildings (stand-alone buildings or individual units within low-rise or high-rise buildings) present lower electric loads and hence possess little negotiation power with utilities [22]. These buildings can, however, interact with an aggregator, which will translate the electricity market incentives into specific control signals for HVAC systems and other electrical devices. A more local incentive for residential buildings could arise from limitations of the electricity distribution grid. This could pose extra demand response incentives on this scale, in order to limit the stress on the distribution grid [23]. In a commercial building context, the magnitude of the electricity demand could be large enough such that direct interaction with the electricity system (on the generation and transmission level) is possible, for example for providing ancillary services [24].

One way of looking at this is from the different levels of operation. The problem can be divided up into roughly six different scales. Each of these has a separate optimization model that could be analyzed and solved. Ideally, the optimization priority would first be with the lowest level and then proceed upward in scale; this is because a system cannot be fully optimized at the higher level if the lower levels are not functioning optimally. The scales of operation can be looked at as:

I. Individual equipment or systems in a building

II. Individual buildings

III. Nanogrid or microgrids

A. Campus, such as a university or complex of buildings (Nanogrids)

B. City (Microgrids)

IV. System (utility, system operator, state or region, etc.)

V. National (country-wide coordinated system)

VI. Continent, world

The various levels of operation were also illustrated to an extent in Fig. 1. 
2.4. Can a smart grid increase vulnerability of the electrical grid and building control systems?

\section{Answer:}

It is natural to think of the potential increased security risk due to the additional connectivity of smart buildings to a smart grid. Control of the smart grid is highly dependent on exploiting smart sensors installed throughout the grid and large communication networks to transfer data between different components. Hence, such heterogeneous communication networks could be used by adversaries to launch various malicious cyber attacks against data integrity $[25,26]$. Recent research efforts have shown that even using existing countermeasure techniques, modern smart grids can be vulnerable to cyber attacks [27]. The integration of smart buildings can increase the potential of cyber attacks at the distribution level, where the current security tools are no longer useful due to the number of users, the limitation of computational power of smart building control systems and their data access limitation. Therefore, smart building control units could become easy targets for adversaries to launch malicious attacks to decrease smart building control system functionality.

Given the relentless increase in size of the smart grids with high penetration of distributed generation and the dominant tendency toward making building energy usage smart, traditional centralized grid control is no longer effective due to the amount of needed data processing, communication bottlenecks and exponential increase in computational burdens of optimization due to the growing control problem dimension. Therefore, there is a movement from conventional hierarchical control to distributed, multi-agent control. Implementation of this distributed control may, however, increase grid vulnerability due to the additional points of entry. Therefore, new secure communication technology and topologies should be investigated. Also, new efficient cyber-attack detection and response mechanisms are needed to prevent possible threats to smart grid integrity.

The communication between buildings and grids may increase the vulnerability of smart buildings to data integrity attacks, and therefore it is essential to develop reliable and fast cyber attack detection tools to prevent possible cyber attacks against building control system. However, it is very important to understand that buildings and the grid were already connected to cyberspace before the onset of a smart grid and smart building technologies. Connection of buildings and their systems to the smart grid does open up more potential avenues for intrusion, but management of these risks is similar to managing the risks for the other Internet connection points.

\subsection{Can integration of a smart grid and smart buildings improve grid stability and resiliency?}

\section{Answer:}

The integration of efficient distributed renewable energy system (RES) into smart grids has led to a decrease in transmission losses and (potentially) the need for installing new transmission lines. However, the stochastic and intermittent nature of wind and solar RESs, has a significant impact on power system stability. Due to the fluctuation of the distributed power generated by RESs, and the challenges to predict available wind and solar generation, even for the day ahead, in a modern smart grid stability analysis the presence of uncertainty and randomness of RES power has become a challenging task. Therefore, making smart grids robust against these uncertainties can be considered as one of the biggest challenges facing reliable operation of future power grids.

Smart buildings can provide a sustainable solution to the increasing complexity of smart grids. Smart buildings are not only energy consumers, but they can also now be prosumers with onsite energy generation systems using solar and perhaps wind. These aspects altogether help smart buildings contribute to improving stability of smart grids by smoothing out the fluctuating loads and distributed power generation of the grid. Smart buildings can be designed to store thermal or electrical energy, which directly helps improve grid stability. Although demand response has typically been thought of as a measure to reduce consumption during periods of high peak demand, as the fraction of intermittent RESs increases, there will be a need to also manage for times with peak energy supply from these systems. This is becoming more common in some locations, such as parts of Europe as well as some regions of the United States.

Demand response can be one of the more important features of smart buildings. This is generally done in response to electricity price signals. However, much of a building load consists of consumption points that generally have two modes of operation (on or off) - examples include most lighting and appliances. Therefore, the response of the smart buildings to electricity price tends to be a discrete function. Given that a considerable grid load is for buildings, simultaneous participation of these loads can possibly lead to frequency oscillations and frequent discontinuity in smart building services.

Smart buildings have the ability to play a critical role in stabilizing the grid by optimizing power consumption. In building control level, model predictive based controllers (MPC) have been recently proposed as powerful tools to optimize the operation of smart buildings, to improve human comfort and improve grid stability [28,29]. Recently, a "Ten Questions" paper was published that covered in detail how MPC can improve the energy efficiency of buildings [30]. The accuracy of MPC-based smart building controllers is very much dependent on accurate prediction of factors affecting energy consumption (such as weather, occupancy patterns, or specific characteristics of the building and system equipment), energy prices and other related factors such as the impact on building occupants.

Integration of the smart grid and smart buildings is not just a technical problem, but also an economic one. Pricing mechanisms are typically effective in balancing supply and demand. Thus, the combination of a smart grid and real-time pricing of electricity is necessary to ensure smart operators adjust a building's demand for electricity to account for the current market situation. The smart grid will be a technical marvel, but economic failure, if the electricity market continues to shield consumers from the reality of supply and demand.

2.6. What can or should be done to legacy HVAC control systems to allow them to interact with a smart grid?

\section{Answer:}

Legacy building control systems vary widely in terms of complexity, vintage, capabilities, and achieved performance, and in individual buildings have often evolved and hybridized organically. They also present a significant barrier to the rapid and full-scale market penetration of smart buildings. Their main role in the past has been that of maintaining temperature setpoints according to predefined schedules, without predictively considering building internal requirements or external information such as weather patterns, grid stress, or utility pricing information. When building operational data is trended, it often remains on-site and is commonly highly underutilized. The proprietary nature of legacy controls limits both their integration and communication capabilities, which are required for effective smart grid participation.

For large commercial buildings, communication standards such as ASHRAE's BACnet overcome many of the hurdles of proprietary 
building communication protocols, allowing many vendors' hardware components to coexist in a building. In addition, proprietary software solutions based on Java enable the connection and integration, thus interoperability, of control devices communicating in BACnet, ModBus, LonTalk, or OPC utilizing Java Application Control Engine (JACE) controllers to gain insight and active control of several geographically dispersed buildings and potential integration with a smart grid aggregation entity. As an illustration, it is technically possible, and practiced by a building-to-grid startup company [31], to compute optimal global temperature setpoints that minimize expected operating cost or achieve desired demand reduction targets on a cloud platform and communicate these with the building in real time using such an interoperability approach. New technology introduced recently can allow for legacy pneumatic thermostats to be wirelessly connected to building control systems, thus giving control functionality similar to modern direct digital control.

A key barrier to the transition of legacy buidings is the cost of upgrading building control system(s). Few small to medium sized buildings are equipped with a centralized BEMS, impeding smart grid functionality through methods described in this article. Instead, for these legacy buildings, smart grid control features are increasingly offered at the component and subsystem level. For example, rooftop units (RTUs) serving the vast majority of the North American conditioned building floor area are seen as ready targets for intelligence enhancements. A specialized technology provider [32] offers a kit concept, which can be installed by an average HVAC technician in 5-6 h per unit. It saves energy by converting from constant air volume to multi-speed airflow using a variable frequency drive with resulting substantial fan energy savings, and provides only the amount of ventilation needed for proper indoor air quality based on current occupancy level. This retrofit kit also will do predictive economizing; when ambient temperatures are cool enough additional outside air is brought in to anticipate future cooling needs. Real-time oversight and control is maintained via a dedicated automation system through cellular connection, allowing users to access their equipment for control changes or fault detection from their smart phones or laptops. With this connectivity, they can provide automatic demand response (via the OpenADR v2.0b protocol) and can provide load shaping leveraging the building's passive thermal capacitance. Yet another vendor offers RTUs equipped with active ice-based thermal energy storage responding to utility DR signals, critical peak pricing, and offering load shaping capabilities.

The emerging market for stationary battery storage will also bring smart grid capability into small and medium buildings. Other ways to integrate smart grid controls into end use devices include large heat pump domestic hot water heaters and advanced LED lighting retrofits where additional wireless sensing and grid communications are seamlessly integrated with the lighting retrofit.

Developing methods and algorithms to coordinate building operations in a manner that adequately serves users, factoring in the capabilities of the existing control systems, while improving electric grid efficiency and renewable resource utilization, is also needed. Approaches have often explored top-down strategies where lower-level devices are directed by higher-level coordinating entities. Strategies often also assume a set of desirable objectives is available from the utility or grid operator that can in some way inform the desired response. Conversely, distributed intelligence approaches seek to achieve similar objectives by equipping local entities with autonomy and problem solving capabilities. In this context, transactive control and coordination is an emerging and potentially transformational concept of distributed intelligence within the context of the building operations and integration of buildings with the electric grid. In a transactive control framework, entities enter into mutually beneficial contracts to provide and procure resources and services [33]. Entities, or transactional nodes, can be anything from an individual device to groups of complex systems. A simple example of such a network is a smart building thermostat that communicates the prices a customer is willing to pay for the various quantities of energy that are needed to achieve different building comfort levels, and a utility that communicates the quantity of energy it is willing to supply at various prices. An equilibrium price is settled upon, the thermostat implements the interior temperature corresponding to the agreed upon price, and the utility supplies the associated quantity of energy. Such strategies and devices mentioned here can be implemented on a piecemeal basis in a building without centralized BEMS or with legacy controls to achieve partial integration with a smart grid.

One anticipated benefit of the transaction-based controls framework is that complex, large-scale problems, that may be difficult to address from a centralized intelligence perspective, can be reduced to numerous simpler decisions executed between individual nodes. Bringing transactive control and coordination to fruition ultimately requires increasing the intelligence of each transactional node, since each entity participating must be able to determine its value of providing and purchasing services and products. Moreover, each node must also be able to execute control actions resulting from market contracts.

In this context, the recently released ANSI/ASHRAE/NEMA Standard 201-2016 [14] provides a common basis for electrical energy consumers to describe, manage, and communicate about electrical energy consumptions and forecasts, ensuring compatibility with the smart grid for current and future technologies; effectively extending the idea of BACnet for building level communication to building-to-grid integration.

\subsection{How can public policy encourage the interaction of a smart grid and smart buildings?}

\section{Answer:}

Policy can do a lot of things to better connect smarter grids and buildings. Public policies operate directly and indirectly to promote interaction between smart grids and smart buildings, from interventions directly affecting deployment of smart grid and smart building technology, the altering of economic and regulatory landscapes, or indirectly influence smart grid-building interactions.

Interventions in grid infrastructure and operations offer the most direct way to advance the smart grid vision. Most straightforwardly, this involves public investment in and subsidized deployment of new smart grid infrastructure. Spending large sums on smart grid investments, as the U.S. did several years ago with federal stimulus funds [34], gives smart buildings a smarter grid to connect to. Although additional public spending may not overcome barriers to deploying upgraded power systems [35,36], new regulatory approaches hold the best promise for promoting more smart grid-building interactions. Demand response programs and shifts to dynamic electricity pricing gives consumers and others the opportunity to benefit from smart building investments [37-39]. Going further, policies that promote distributed generation, and distributed storage, for example with plug-in cars [40,41], increase the benefits of smart building capabilities to capture the value created [42]. Feed-in tariffs and net-metering rules provide incentives to deepen the interaction between smart buildings and the grid, although they are not without concerns about optimal incentive size and equity [43]. Other regulatory shifts can support smart grid development by rewarding innovating utilities [44], allowing for societal benefits and more flexible cost recovery $[45,46]$, and other decoupling efforts $[46,47]$. Costly new 
investments will require policymakers and regulators to carefully set rules to appropriately allocate costs, share risks, and align incentives $[44,48]$. As with other aspects of outmoded regulatory frameworks, a smart grid vision and upgraded transmission infrastructure depends on shifting planning and regulatory authority beyond state control [38].

Policymakers can also directly promote smarter grid-building interactions by directly supporting the smart building side. Public building construction and procurement rules can account for a large share of the sector and catalyze broader changes rippling across the industry. Many green certified (e.g., LEED) buildings of the last decade are government-owned [49] and, like the effect on green buildings, public procurement rules favoring smart buildings can help smarter technologies diffuse and reach critical mass in the market [50]. Conventional regulatory tools like buildings codes and appliance efficiency standards remain available to push greater adoption of smart buildings engaging the grid.

The past couple of decades have seen the growth in policy and regulatory drivers toward smart buildings and their interaction with a smart grid. These include, for example, incentives or requirements for green building practices, participation in demand response programs, building energy use reporting, net metering for on-site renewable energy systems, etc. Indirect approaches may have the largest impact by pervasively altering incentives for grid and building users. Building out the smarter energy services ecosystems and marketplaces entails better coordination at multiple levels of government and overhauling outdated regulatory frameworks $[38,46,48]$. Policies that reduce financing costs for infrastructure investments [48], subsidize smarter designs [51], or give tax advantages to smart grid assets or renewables can accelerate these trends [48]. A variety of policy instruments - from 'nudges' to subsidies to mandates - can induce customers and investors to better utilize smart building capabilities, especially when facing psychological or behavioral constraints [44,52]. Ultimately, policies that work to minimize carbon emissions - particularly carbon pricing policies - may advance smart grid-building interactions most of all by ensuring prices reflect full social costs, albeit for environmental reasons. Continuing the broader trends toward greater intermittent generating capacity in renewables and to more distributed generation will increase returns to smart grid investments [37,38] and interactions with smart buildings.

Progress here depends on promoting innovative platforms to create societal value as well as protecting against unintended consequences. Societal gains far outweigh gains to individual building owners when buildings are integrated with the grid. Any incentives that utilities provide will be small compared to the societal gains. Unleashing market forces to attract the investment and realize the promise of smarter grid-building integrations means new platforms for delivering energy services [53]. Policymakers can encourage the development of these platforms. This means promoting and enforcing rules for transparency and privacy for consumers, while regulators should carefully monitor antitrust concerns that arise. Policymakers can also help establish interoperability standards [54]. Maintaining regulatory flexibility here not policymakers or regulators picking winners - is vital in the decentralized and dynamic ICT [54]. Policymakers can proactively address customer resistance and engage "smart users" to realize greater change [55]. Policymakers may proactively address vulnerable groups and those adversely affected by these changes, especially resulting from changes in pricing systems [56,57].

\subsection{How can society encourage participation in smart grid and demand response programs?}

\section{Answer:}

There are both technical and non-technical barriers to fully developing an integrated smart grid and smart buildings. Previous questions in this article have addressed technologies that are making this possible and how public policy measures can be adjusted to encourage its adoption. However, just because the technologies are in place does not guarantee they will be utilized. Humans operate from a blend of rational and social motivations [58]. The rational dimension underlies much of economic analysis, because it assumes that humans act in self-interest, whereas the social side recognizes that culture and society are influenced by the behaviors and opinions of others. These conflicting goals are also presented as a battle between prices and perceptions [59]. Prices align with rational action and perceptions influence social behavior. It must also be recognized that there are different scales and structures involved; an owner of a single-family residential unit will have different motivations and value structures compared to the owner/manager of a large commercial building or complex of buildings.

A rationalist would argue that enterprises will participate in the smart grid and demand response systems when it is in their selfinterest. In other words, they can reduce costs by avoiding peak prices or participating in demand response events. However, enterprises like some degree of certainty so they can plan their activities to minimize costs. Consequently, they need access to information systems, internally or externally provided, that can predict electricity prices or demand response events for several days ahead on a rolling horizon. Agile organizations can then monetize their flexibility by planning to avoid high electricity prices. This of course depends on whether future energy prices are known with certainty (for example with a day-ahead guaranteed real-time price schedule) or simply a forecast with its associated uncertainty. The response may be different between these two scenarios. The technical community can help identify and implement such opportunities. For example, the campus district chiller project at the University of Georgia aims to reduce electricity demand by treating the piping connecting buildings as a built-in cold water storage that can be deployed to help flatten peak cooling demand [60].

On the social side, management scholars have learned that in order to survive organizations must conform to the social norms of their environment [61]. Like humans, organizations are influenced by the actions of their peers. As the American Psychological Association observed in 2007, "When it comes to persuading people to conserve energy, the message "everybody else is doing it" works better than trying to appeal to people's sense of social responsibility." As well as arising from imitation, new norms can be coerced [61]. Powerful buyers can force their suppliers to adopt new behaviors. For imitation to work however, adoption of the smart grid and demand response is dependent on finding some successful first adopters and publicizing their success so that others mimic their actions and a new institutional norm is established.

On the coercive side, most corporations know that driving costs out of the supply chain by pressuring suppliers to adopt more efficient processes makes an enterprise more cost competitive. Thus, self-interest should ignite the diffusion of cost-efficient practices spreading to suppliers.

The initiative can also come from demand response aggregators, whose sole product is to activate the flexibility at the electricity demand side. Instead of a building manager calculating price forecasts and putting time and effort in learning the particularities of the electricity market, the aggregator performs this work. The aggregator might take control of a building's HVAC for a limited number of hours in the year, for typically a predetermined monetary compensation for the building owner and with preset limits on what is done. In this context, it is up to the aggregator to actively 
search and contact building owners for participation in demand response. In other situations, the motivator may not be monetary but rather other reasons, such as a desire to minimize the carbon intensity of electricity consumed.

\subsection{What strategies can direct the behavior of building owners, operators and occupants (or operators, or occupants) toward an improved interaction with a smart grid?}

\section{Answer:}

A large investment is now being made to transition to a smart grid; yet, the energy saving and financial benefits of this infrastructure will only reach their full potential with careful consideration of the human dimension, mostly with the behavioral component. When building systems adjust based on connections with a smart grid, such as when implemented demand response measures, careful concern must be taken to avoid a negative impact on occupants or their perception of the overall indoor environmental quality.

We propose a dual approach to adjust behaviors towards an improved interaction with a smart grid:

(1) push comparative data about similar buildings' energy use (along with the associated energy cost and $\mathrm{CO}_{2}$ emissions) over time; and

(2) disaggregate where possible such comparative data at the building equipment and individual zone levels to identify reduction targets.

By supplying comparative information to building owners, operators, and business occupants, they can benchmark their efforts and be enticed to set new targets for energy reduction. Comparative information can also help alleviate any negative perceptions the occupants may have when adjustments are made, such as changes in temperature setpoints in the building zones. When it comes to persuading people to conserve energy, peer pressure works best, according to a landmark behavioral science experiment [62]. Contrary to conventional wisdom, which suggests that financial gains, environmental concerns, or the desire to be a good citizen are the main motivators to change behaviors, comparative information is more likely to influence one to behave in a particular way.

In the residential market, many households now receive a "home energy report" (HER), which allows them to compare their energy usage with that of their neighbors. New online technologies are coming that will allow customers to better use this information and reduce overall energy consumption. Field experiments to understand the impact of HER have corroborated the savings potential $[63,64]$, and both studies confirm a 1.2 to $2.1 \%$ reduction in energy consumption on households receiving such reports. While these percentages do not seem that large, the total energy consumed in the residential housing market is roughly the same as with commercial building, so the overall potential is large.

More recently, the focus has moved outside the household, and researchers have investigated the hotel industry, which is greatly impacted by large costs for lighting and HVAC. Chang and his colleagues recently demonstrated that guest-driven electricity reduction can be achieved in such contexts as well [65]. From these studies, we suggest that business operators who have knowledge about the energy consumption of not only their buildings, but also of others comparable in type and size, would be more likely to look into how to reduce their energy usage if they fare poorer than their peers. Thus, assuming that a general ethos favoring sustainability emerges, comparative data are likely to create a virtuous cycle of diminishing environmental impact [66].
A second approach to change behaviors towards an improved interaction with a smart grid involves the disaggregation of data at the building, equipment and individual zone levels to identify reduction targets [66]. Disaggregation refers to the breaking of information into its constituent parts. It involves sensors and statistical approaches for extracting finer level data from an aggregate, or whole-building, energy signal. Again, starting with a household context, a consumer who elects to run a dryer for a few hours or leaves an entire floor lit for the day knows little about the energy usage associated with these choices. Similarly, if this consumer is interested in running some appliances when electricity comes primarily from sustainable sources, they typically lack this information on a dynamic basis. This consumer's monthly electricity bill, if it is like the majority of households, does not include information disaggregated by devices, location, or time. That is because electricity consumption is typically measured for large units, such as an entire house or for a month. On the commercial market, if electricity consumption is disaggregated so as to know the energy usage associated with individual components (e.g., a particular office or even a particular device), building owners and occupants would better understand the impact of their behaviors on the environment $[67,68]$. Indeed, such descriptive datasets have been shown to inform and empower a wide variety of energy stakeholders, from building operators to utilities and policy makers [69]. Moreover, disaggregate data can also enable personalized and automated recommendations [70], such as indicating which rooms within a given building use the most for HVAC and lighting relative to their actual occupancy.

The two approaches discussed previously are relatively easy in concept to implement. By combining them, we argue, they provide a powerful environmental nudge, that is, a way to influence building stakeholders to do the right thing without compromising their freedom of choice [71]. Indeed, comparative and disaggregate data, when available, open the door to friendly competition among floors, departments, and units in a commercial setting, and such a gaming context is prone to make change happen [72].

\subsection{What are the key research and development needs to speed future implementation of a smart grid and smart buildings?}

\section{Answer:}

It should be apparent from the prior questions and answers that control capability and data exchange are fundamental keys to the integration of a smart grid and smart buildings. Automated demand response (ADR) effects changes in electric usage implemented directly or indirectly by end-use customers/prosumers from their normal consumption and injection patterns in response to certain signals [73]. The resultant load change modifies the electric load profile e.g. of electrically driven air-conditioning systems by decoupling in time the demand for electrical demand and thermal power to yield operational benefits at the electric system level [74]. More study is needed on methods to optimize the response from the perspective of building operations, occupant impacts, and overall benefit to the grid.

Prime among the methods which can shift electricity demand in time with minimal to no impact on process quality is the thermally relatively inert process of providing heating or cooling [75]. Wang et al. [76] and Dupont [77] show that automatic control achieves higher degrees of response than manual programs. Smart thermostats have drastically increased their market share in recent years and continued growth is forecast [78,79]. Apart from improving energy efficiency, some of these Internet connected smart thermostats already perform peak shaving while maintaining thermal comfort [80]. Maybe these smart thermostats could do more than occasional peak shaving? 
ADR programs require cost effective control methods and equipment, and additional research and development is needed to reduce first cost for equipment and software and operating cost to run and maintain the system, while increasing system robustness.

Demand flexibility expands the capability of ADR programs by allowing demand to respond continuously to changing market conditions through price signals or other mechanisms to achieve a continuous integration of building operations with the electric grid system. A recent report on the economic value of demand flexibility by the Rocky Mountain Institute [81] states that the potential gridlevel cost savings from widespread demand flexibility deployment may be USD 9 billion per year in traditional investments for generation, transmission, and distribution. Additional costs of up to USD 3 billion per year can be avoided by controlling the timing of a small fraction of these appliances' energy demands to optimize for dynamic energy prices, and USD 1 billion per year from providing ancillary services to the grid. Yet, how can such continuous flexibility in demand be attained?

This question is investigated by Patteeuw, Henze, and Helsen [82], in which an integrated modeling approach is developed in which the electricity generation system is concurrently optimized with the operation of the residential heat pumps for the case of Belgium. The integrated formulation shows significant reductions in total operational costs from better part-load operation of the power plants, a reduction in start-up and ramping of power plants and the reduction in curtailment of electricity generation from RES. They also indicate that employing peak energy supply from RESs at times when RESs overshoot electricity demand has the potential to lower carbon emissions associated with building energy use, and this is an area needing further investigation for optimization routines. In a second step, multiple decentralized control methods, including dynamic pricing schemes, are assessed and compared with the integrated optimization. Surprisingly, it is revealed that dynamic price profiles as signals can lead to unintended adverse effects and system instability. Thus, broadcasting day-ahead or real-time energy prices may prove to be risky. With the increasing share of smart thermostats, which can act upon such dynamic price profiles, dangerous artifacts of greedy control actions could occur in the near future. A central determination of a load profile for all buildings to follow individually, known as load shaping, proves to be a far better option. Yet, would such load shaping be beneficial in a cooling dominated context as well? This area also needs further study.

Corbin and Henze $[83,84]$ address this question and explore the potential for large-scale aggregations of thermostaticallycontrolled building electrical loads to actively shape distribution feeder electric demand, with objectives of reducing peak demand and system ramping as well as improving electric system utilization by increasing system load factor using a distributed but directed model predictive control scheme. Cases in which high penetration levels of distributed roof-top photovoltaic and utility scale wind generation are investigated suggest that the load shaping methodology is very effective at providing demand flexibility at time scales shorter than two or three hours. At longer time scales, however, flexibility is limited by the thermal storage effectiveness of the residential building envelope, and to a large extent, the amount of load being shifted relative to the total cooling demand.

Research is needed to develop methods that will optimize the energy consumption in conjunction with human factors, such as the perceived thermal comfort of the occupants. Research is also needed to establish procedures for dynamically defining effective target load shapes for smart thermostats to drive each building demand such that the electric grid system benefits are optimally balanced with customers' desire for reliable and low-cost electricity and the transition towards a sustainable and decarbonized society through RES integration, in hopes that the substantial savings that are estimated to be possible [81] can be achieved.

Research scholars can prime both rational and social decision making, for example in developing methods for forecasting electricity prices and working to identify and aid potential first adopters in implementing new procedures for agility-based cost reduction by adjusting activities to prices. Modeling forecast of weather and electricity prices must be accurate enough such that system operators, aggregators and building owners can make financially responsible decisions. Uncertainty about price forecasts can be a barrier to implementation. There is also a need to publish these ideas beyond just academic and technical journals, for example by speaking at practitioner conferences, teaching students, and writing case studies.

Nurturing the adoption of the smart grid and demand response systems requires a holistic approach. Designers, managers and researchers of the built environment can investigate and develop the necessary technology, such as electricity pricing forecasting and automated controls for agile reaction to pricing peaks. However, acknowledgement is needed that technologies are embedded in a socio-technical system, thus, study is also needed on the impact on adoption and diffusion of new technologies and their related actions on society. Market adoption will be improved by additional large scale demonstration projects and case studies. Technological innovation is clearly required, but so is organizational change to take advantage of the innovation.

\section{Expertise of the authors}

Drs. Lawrence, Watson, Mohammadpour and Boudreau are the key collaborators in the Energy Informatics research group at the University of Georgia. Drs. Watson and Boudreau published a seminal book on the concept of Energy Informatics in 2010. Dr. Mohammadpour's current work focuses on the modeling and advanced control of the smart grids operating in the presence of stochastic loads and renewable energy sources. Dr. Lawrence currently has several research projects funded by Georgia Power for studying how demand response can be integrated into the existing building and systems controls at the University of Georgia campus grid, and also conducts workshops and seminars for ASHRAE on the topics of smart buildings and sustainability in the built environment. Dr. Henze is a professor of Architectural Engineering at the University of Colorado - Boulder and researches model predictive control and data analytics for integration of building energy systems with the smart grid. Dr. Helsen is Professor in the Applied Mechanics and Energy Conversion Section at KU Leuven in Belgium and an expert in the integration of renewable energy systems with buildings and the integrated optimal design and control of building systems. Dr. Noonan is the Director of Research at the Indiana University Public Policy Institute School of Public and Environmental Affairs and has published papers in relevant areas such as the economic and social dimensions for adoption of energy efficient behaviors. Shanti Pless is a senior research engineer at the National Renewable Energy Laboratory and has written more than 50 journal articles, industry publications, guidebooks, and technical reports related to energy efficiency and zero-energy buildings.

\section{Acknowledgements}

Supporting for research in this topic at the University of Georgia was provided in part by Georgia Power and the Southern Company.

\section{References}

[1] U.S. Department of Energy, 2011 Buildings Energy Data Book, Building 
Technologies Program, Energy Efficiency and Renewable Energy, U.S. Department of Energy, 2012. March 2012. Available Online at: http:// buildingsdatabook.eren.doe.gov/.

[2] F. Pigni, G. Piccoli, R.T. Watson, Digital Data Streams: creating value from the real-time flow of big data, Calif. Manag. Rev. 58 (3) (2016) 5-25.

[3] I. Georgievski, V. Degeler, G.A. Pagani, T.A. Nguyen, A. Lazovik, M. Aiello, Optimizing energy costs for offices connected to the smart grid, IEEE Trans. Smart Grid 3 (4) (2012) 2273-2285.

[4] M. Pipattanasomporn, Multi-agent systems in a distributed smart grid: design and implementation, in: IEEE Power Systems Conference and Exposition (PSCE’09), Seattle, Washington USA, 2009.

[5] A. Kantamneni, L.E. Brown, G. Parker, W.W. Weaver, Survey of multi-agent systems for microgrid control, Eng. Appl. Artif. Intell. 45 (2015) 192-203, http://dx.doi.org/10.1016/j.engappai.2015.07.005.

[6] L.A. Hurtado, P.H. Nguyen, W.L. Klinig, Smart grid and smart building interoperation using agent-based particle swarm optimization, Sustain. Energy, Grids Netw. 2 (2015) 32-40.

[7] L. Klein, J. Kwak, F. Kavuya, B. Becerik-Gerber, P. Varakantham, M. Tambe, Coordinating occupant behaviour for building energy and comfort management using multi-agent systems, Autom. Constr. 22 (2012) 525-536.

[8] T. Labeodan, K. Aduda, G. Boxem, W. Zeiler, On the application of multi-agent systems in buildings for improved building operations, performance and smart grid interaction - a survey, Renew. Sustain. Energy Rev. 50 (2015) 1405-1414.

[9] D. Kolokotsa, D. Rovas, E. Kosmatopoulos, K. Kalaitzakis, A roadmap towards intelligent net zero- and positive energy buildings, Sol. Energy 85 (2011) 3067-3084.

[10] W. Zeiler, G. Boxem, Smart grid - building energy management system: an ontology multi-agent approach to optimize comfort demand and energy supply, ASHRAE Trans. 119 (2) (2013) 1-8.

[11] ASHRAE, ANSI/ASHRAE Standard 62.1-2016 Ventilation for Acceptable Indoor Air Quality, American Society of Heating, Refrigeration and Air Conditioning Engineers, Atlanta, Georgia, 2016.

[12] OpenADR Alliance, OpenADR 2.0 Profile Specification, 2015. Document Number 2012091201, Updated 11-17-2015, http://www.openadr.org/ specification-download.

[13] E.-K. Lee, Advancing building energy management system to enable smart grid interoperation, Int. J. Distrib. Sens. Netw. 2016 (2016), http://dx.doi.org/ 10.1155/2016/3295346. Article ID 3295346, 12 pages.

[14] ASHRAE, ANSI/ASHRAE/NEMA Standard 201-2016 Facility Smart Grid Information Model, American Society of Heating, Refrigeration and Air Conditioning Engineers, Atlanta, Georgia, 2016.

[15] U.S. Green Building Council, LEED v4 for Building Design and Construction, 2016. Washington, D.C. Updated April 5, 2016.

[16] International Code Council, International Green Construction Code, IgCC 2015, 2015. Washington, DC, http://www.iccsafe.org/codes-tech-support/codes/ 2015-i-codes/igcc/.

[17] R.K. Akikur, R. Saidur, H.W. Ping, K.R. Ullah, Comparative study of stand-alone and hybrid solar energy systems suitable for off-grid rural electrification: a review, Renew. Sustain. Energy Rev. 27 (2013) 738-752.

[18] G. Strbac, Demand side management: benefits and challenges, Energy policy 36 (12) (2008) 4419-4426.

[19] N.J. Kelly, P.G. Tuohy, A.D. Hawkes, Performance assessment of tariff-based air source heat pump load shifting in a UK detached dwelling featuring phase change-enhanced buffering, Appl. Therm. Eng. 71 (2) (2014) 809-820.

[20] D. Patteeuw, G.P. Henze, L. Helsen, Comparison of load shifting incentives for low-energy buildings with heat pumps to attain grid flexibility benefits, Appl, Energy 167 (2016) 80-92.

[21] D. Patteeuw, K. Bruninx, A. Arteconi, E. Delarue, W. D’haeseleer, L. Helsen, Integrated modeling of active demand response with electric heating systems coupled to thermal energy storage systems, Appl. Energy 151 (2015) $306-319$.

[22] L. Gkatzikis, I. Koutsopoulos, T. Salonidis, The role of aggregators in smart grid demand response markets, Sel. Areas Commun. IEEE J. 31 (7) (2013) $1247-1257$

[23] N. Leemput, F. Geth, B. Claessens, J. Van Roy, R. Ponnette, J. Driesen, A case study of coordinated electric vehicle charging for peak shaving on a low voltage grid, in: Innovative Smart Grid Technologies (ISGT Europe), 2012 3rd IEEE PES International Conference and Exhibition on, 2012, October, pp. 1-7.

[24] G.S. Pavlak, G.P. Henze, V.J. Cushing, Optimizing commercial building participation in energy and ancillary service markets, Energy Build. 81 (2014) $115-126$.

[25] W. Wang, Z. Lu, Cyber security in the smart grid: survey and challenges, Comput. Netw. 57 (5) (2013) 1344-1371.

[26] T. Baumeister, Literature Review on Smart Grid Cyber Security, Technical Report, University of Hawai'I, 2010, http://csdl.ics.hawaii.edu/techreports/1011/10-11.pdf.

[27] Y. Liu, N. Peng, M.K. Reiter, False data injection attacks against state estimation in electric power grids, ACM Trans. Inf. Syst. Secur. TISSEC 14 (1) (2011) 13.

[28] Y. Ma, F. Borrelli, B. Hencey, A. Packard, S. Bortoff, Model predictive control of thermal energy storage in building cooling systems, in: Decision and Control, 2009 Held Jointly with the 2009 28th Chinese Control Conference. CDC/CCC 2009. Proceedings of the 48th IEEE Conference on, 2009, pp. 392-397.

[29] Y. Ma, A. Kelman, A. Daly, F. Borelli, Predictive control for energy efficient buildings with thermal storage, IEEE Control Syst. Mag. 32 (1) (2012) 44-64.
[30] M. Killian, M. Kozek, Ten questions concerning model predictive control for energy efficient buildings, Build. Environ. 105 (2016) 403-412.

[31] C.D. Corbin, G.P. Henze, P. May-Ostendorp, A model predictive control optimization environment for real-time commercial building application, J. Build Perform. Simul. 6 (3) (2013) 159-174.

[32] I. Doebber, J. Dean, J. Dominick, G. Holland, RM12-2703 Advanced Rooftop Unit Control Retrofit Kit Field Demonstration, 2014. NREL Technical Report NREL/TP-5500-61072, http://www.nrel.gov/docs/fy14osti/61072.pdf.

[33] S. Somasundaram, R.G. Pratt, B.A. Akyol, N. Fernandez, N. Foster, S. Katipamula, E.T. Mayhorn, A. Somani, A. Steckley, Z.T. Taylor, Transactionbased Building Controls Framework, in: Reference Guide, vol. 1, Pacific Northwest National Laboratory, Richland, Washington, 2014. Technical Report PNNL-23302.

[34] US Department of Energy, Smart Grid Legislative and Regulatory Policies and Case Studies, 2011. https://www.eia.gov/analysis/studies/electricity/pdf smartggrid.pdf.

[35] J.E. Aldy, Policy monitor a preliminary assessment of the american recovery and reinvestment act's clean energy package, Rev. Environ. Econ. Policy 7 (1) (2013) 136-155.

[36] G.E. Metcalf, Taxing Energy in the United States: Which Fuels Does the Tax Code Favor, The Manhattan Institute, New York, 2009.

[37] B. Hamilton, M. Summy, Benefits of the smart grid [in my view], Power Energy Mag. IEEE 9 (1) (2011), 104-102.

[38] P.L. Joskow, Creating a smarter US electricity grid, J. Econ. Perspect. 26 (1) (2012) 29-48.

[39] P. Siano, Demand response and smart grids-a survey, Renew. Sustain. Energy Rev. 30 (2014) 461-478.

[40] Z. Wang, L. Wang, A.I. Dounis, R. Yang, Integration of plug-in hybrid electric vehicles into energy and comfort management for smart building, Energy Build. 47 (2012) 260-266.

[41] O. Erdinc, Economic impacts of small-scale own generating and storage units and electric vehicles under different demand response strategies for smart households, Appl. Energy 126 (2014) 142-150.

[42] F. Rahimi, A. Ipakchi, Demand response as a market resource under the smart grid paradigm, Smart Grid IEEE Trans. 1 (1) (2010) 82-88.

[43] A. Brown, J. Bunyan, Valuation of distributed solar: a qualitative view, Electr. J. 27 (10) (2014) 27-48.

[44] World Economic Forum (WEF), Accelerating Successful Smart Grid Pilots, 2010. WEF Report. Available from: http://www3.weforum.org/docs/WEF_EN_ SmartGrids_Pilots_Report_2010.pdf.

[45] The Economist, Wiser Wires-information Technology Can Make Electricity Grids Less Wasteful and Much Greener, 2009. Businesses have Lots of Ideas and Governments are keen, but Obstacles Remain. Online Article. Available from: www.economist.com.

[46] World Economic Forum (WEF), Accelerating Smart Grid Investments, 2009 WEF Report. Available from: http://www.weforum.org/reports/acceleratingsmartgrid-investments.

[47] Institute for Electric Innovation, State Electric Efficiency Regulatory Frameworks, 2014. IEI Report, December 2014.

[48] G.E. Metcalf, Taxing Energy in the United States: Which Fuels Does the Tax Code Favor, The Manhattan Institute, New York, 2009.

[49] D.C. Matisoff, D.S. Noonan, A.M. Mazzolini, Performance or marketing benefits? the case of LEED certification, Environ. Sci. Technol. 48 (3) (2014) 2001-2007.

[50] T. Simcoe, M.W. Toffel, Government green procurement spillovers: evidence from municipal building policies in California, J. Environ. Econ. Manag. 68 (3) (2014) 411-434.

[51] A. Chegut, P. Eichholtz, N. Kok, The Price of Innovation: an Analysis of the Marginal Cost of Green Buildings, 2015. Working paper. Available from: http://www.corporate-engagement.com/files/publication/CEK_Cost_102315. pdf.

[52] H. Allcott, S. Mullainathan, D. Taubinsky, Energy policy with externalities and internalities, J. Public Econ. 112 (2014) 72-88.

[53] V. Giordano, G. Fulli, A business case for Smart Grid technologies: a systemic perspective, Energy Policy 40 (2012) 252-259.

[54] H. Farhangi, The path of the smart grid, Power energy Mag. IEEE 8 (1) (2010) $18-28$.

[55] M. Goulden, B. Bedwell, S. Rennick-Egglestone, T. Rodden, A. Spence, Smart grids, smart users? The role of the user in demand side management, Energy Res. Soc. Sci. 2 (2014) 21-29.

[56] K. Herter, Residential implementation of critical-peak pricing of electricity, Energy Policy 35 (4) (2007) 2121-2130.

[57] V. Giordano, G. Fulli, A business case for Smart Grid technologies: a systemic perspective, Energy Policy 40 (2012) 252-259.

[58] H.A. Simon, Models of Man: Social and Rational, Wiley, New York, NY, 1957.

[59] R.T. Watson, J. Corbett, M.-C. Boudreau, J. Webster, An information strategy for environmental sustainability, Commun. ACM 55 (7) (2012) 28-30.

[60] T.M. Lawrence, R.T. Watson, Information Systems Needs for Adaptation of Existing Campus Facilities for Automated Demand Response, Erasmus Energy Forum, Rotterdam, Holland, 2016. May 2016.

[61] P. DiMaggio, W. Powell, The iron cage revisited: institutional isomorphism and collective rationality in organizational fields, Am. Sociol. Rev. 48 (2) (1983) 147-160.

[62] R. Cialdini, Understanding and Motivating Energy Conservation via Social Norms, Hewlett Foundation, 2004. 
[63] H. Allcott, Social norms and energy conservation, J. Public Econ. 95 (9-10) (2011) 1082-1095.

[64] I. Ayres, S. Raseman, A. Shih, Evidence from two large field experiments that peer comparison feedback can reduce residential energy usage, J. Law Econ. Organ. 29 (5) (2013) 992-1022.

[65] H.S. Chang, C. Huh, M.J. Lee, Would an energy conservation nudge in hotels encourage hotel guests to conserve? Cornell Hosp. Q. 57 (May 2016) 172-183, http://dx.doi.org/10.1177/1938965515588132.

[66] R.T. Watson, M.-C. Boudreau, Energy Informatics, first ed., Green ePress, 2011. Available on Amazon at: http://www.amazon.com/Energy-Informatics-ebook/ dp/B005HW4MAA.

[67] B. Neenan, J. Robinson, Residential Electricity Use Feedback: a Research Synthesis and Economic Framework, EPRI Technical Update 1016844, Electric Power Research Institute, Palo Alto, CA, 2009.

[68] K. Ehrhardt-Martinez, K.A. Donnelly, J.A. Laitner, Advanced Metering Initiatives and Residential Feedback Programs: a Meta-review for Household Electricity-saving Opportunities, Technical Report E105, American Council for an Energy-Efficient Economy, Washington, DC, 2010.

[69] J. Froehlich, E. Larson, S. Gupta, G. Cohn, M.S. Reynolds, S.N. Patel, Disaggregated end-use energy sensing for the smart grid, IEEE Pervasive Comput. 10 (1) (2011) 28-39.

[70] K.C. Armel, A. Gupta, G. Shrimali, A. Albert, Is disaggregation the holy grail of energy efficiency? the case of electricity, Energy Policy 52 (2013) 213-234, http://dx.doi.org/10.1016/j.enpol.2012.08.062. ISSN 0301-4215.

[71] R.H. Thaler, C.R. Sunstein, Nudge: Improving Decisions about Health, Wealth, and Happiness, Yale University Press, New Haven, 2008.

[72] D. Geelen, D. Keyson, S. Boess, H. Brezet, Exploring the use of a game to stimulate energy saving in households, J. Des. Res. 10 (1-2) (2012) 102-120.

[73] X. He, L. Hancher, I. Azevedo, N. Keyaerts, L. Meeus, J.-M. Glachant, Shift, Not Drift: towards Active Demand Response and beyond, 2013. Technical report European Commission FP7 project THINK. Available at: http://www.eui.eu/ Projects/THINK/Documents/Thinktopic/Topic11digital.pdf.
[74] A. Arteconi, N.J. Hewitt, F. Polonara, State of the art of thermal storage for demand-side management, Appl. Energy 93 (2012) 371-389.

[75] P. Palensky, D. Dietrich, Demand side management: demand response, intelligent energy systems, and smart loads, Ind. Inf. IEEE Trans. 7 (3) (2011) $381-388$.

[76] J. Wang, M. Biviji, W.M. Wang, Lessons learned from smart grid enabled pricing programs, in: Power and Energy Conference at Illinois (PECI), IEEE, 2011, pp. 1-7, http://dx.doi.org/10.1109/PECI.2011.5740488.

[77] B. Dupont, K. Dietrich, C. De Jonghe, A. Ramos, R. Belmans, Impact of residential demand response on power system operation: a Belgian case study, Appl. Energy 122 (2014) 1-10.

[78] M.H. Albadi, E.F. El-Saadany, A summary of demand response in electricity markets, Electr. Power Syst. Res. 78 (11) (2008) 1989-1996.

[79] Grand View Research, Smart Thermostat Market Analysis by Technology (Wifi, ZigBee) and Segment Forecasts to 2022, Technical report, 2015, ISBN 978-168038-513-7, http://www.grandviewresearch.com/industry-analysis/smartthermostat-market.

[80] Y. Matsuoka, Our First Rush Hour Rewards Results, 2013. Online, https://nest. com/blog/2013/07/18/our-first-rush-hour-rewards-results/.

[81] P. Bronski, M. Dyson, M. Lehrman, J. Mandel, J. Morris, T. Palazzi, S. Ramirez, H. Touati, The Economics of Demand Flexibility, Technical Report, Rocky Mountain Institute, 2015.

[82] D. Patteeuw, G.P. Henze, L. Helsen, Comparison of load shifting incentives for low-energy buildings with heat pumps to attain grid flexibility benefits, Appl. Energy 167 (2016) 80-92. http://www.sciencedirect.com/science/article/pii/ S0306261916300162.

[83] C.D. Corbin, G.P. Henze, Residential HVAC as a supply following resource part I: simulation framework and model development, J. Build. Perform. Simul. (2016 (in press)).

[84] C.D. Corbin, G.P. Henze, Residential HVAC as a supply following resource part II: simulation studies and results, J. Build. Perform. Simul. (2016 (in press)). 